\title{
La pseudonymie dans la littérature française. De François Rabelais à Éric Chevillard, études réunies et présentées par David MARTENS
}

\section{Luana Doni}

\section{(2) OpenEdition}

\section{Journals}

\section{Edizione digitale}

URL: https://journals.openedition.org/studifrancesi/12324

DOI: 10.4000/studifrancesi. 12324

ISSN: 2421-5856

\section{Editore}

Rosenberg \& Sellier

\section{Edizione cartacea}

Data di pubblicazione: 1 avril 2018

Paginazione: 184-186

ISSN: 0039-2944

\section{Notizia bibliografica digitale}

Luana Doni, «La pseudonymie dans la littérature française. De François Rabelais à Éric Chevillard, études réunies et présentées par David maRTENS», Studi Francesi [Online], 184 (LXII | I) | 2018, online dal 04 juillet 2018, consultato il 17 novembre 2021. URL: http://journals.openedition.org/studifrancesi/12324 ; DOI: https://doi.org/10.4000/studifrancesi.12324

Questo documento è stato generato automaticamente il 17 novembre 2021.

\section{(c) $(1)$}

Studi Francesi è distribuita con Licenza Creative Commons Attribuzione - Non commerciale - Non opere derivate 4.0 Internazionale. 


\title{
La pseudonymie dans la littérature française. De François Rabelais à Éric Chevillard, études réunies et présentées par David MARTENS
}

\author{
Luana Doni
}

\section{NOTIZIA}

La pseudonymie dans la littérature française. De François Rabelais à Éric Chevillard, études réunies et présentées par David MARTENS, Rennes, Presses Universitaires, 2017, «La Licorne» 123, $345 \mathrm{pp}$.

1 Il volume, introdotto e diretto da David MARTENS, raccoglie gli atti del convegno tenutosi presso l'Università Cattolica di Louvain sul fenomeno della pseudonimia in letteratura attraverso i secoli.

2 Suddiviso in varie sezioni, si apre con l'intervento di Jean-Pierre CAVAILLÉ (Motivations de la pseudonymie dans "Les auteurs déguisés" d'Adrien Baillet, pp. 17-35) che studia il modo in cui il critico seicentesco Adrien Baillet rende conto del fenomeno e della varietà delle motivazioni per cui uno scrittore è portato a adottare uno pseudonimo. L'introduzione di Cavaillé permette di passare dal generale dell'opera di Baillet vista come teoria dello pseudonimo alla specificità delle varie sezioni a cominciare dalla prima: «Doublures Pseudonymiques».

In questa prima parte si cerca di indagare su un particolare aspetto della pseudonimia: l'eteronimia quale attribuzione di un'opera da parte dell'autore reale ad un autore fittizio, suo alter ego. La riflessione di Ariane BAYLE (D'Alcofribas Nasier à François Rabelais: différer et promettre, pp. 45-58) analizza lo pseudonimo scelto da François Rabelais per sancire il suo ingresso in letteratura. Bayle illustra come Alcofribas Nasier non sia solo lo pseudonimo di Rabelais, ma un narratore-commentatore e personaggio dell'opera. 
Nonostante la graduale scomparsa dello pseudonimo a vantaggio dell'identità dichiarata dell'autore, Alcofribas resta pur sempre il nome dato ad una maniera d'essere nella scrittura di cui Rabelais continuerà a preservare il ricordo nella sua opera.

La molteplicità degli pseudonimi usati da Gérard Labrunie, passato alla storia come Gérard de Nerval, è il soggetto della riflessione di Michel BRIX (Beuglant, Gérard, Aloysius, Fritz, Nerval et Cie: Gérard Labrunie et ses doubles, pp. 59-70) che rivela la volontà dell'autore di fornire una varietà di immagini di sé al proprio pubblico, volontà che va di pari passo con il problema identitario, una delle principali tematiche degli scritti di Nerval.

pesso inverso, quello che va invece dalla molteplicità all'unità, è quello adottato dai componenti del movimento dadaista di cui si occupa l'articolo proposto da Eddie BREUIL ( $D u$ nom dans Dada, pp.71-81). Un'unità però illusoria, quella implicata dall'adozione di un "nome anonimo" quale Dada, giacché ogni membro del gruppo avanguardista, da Berlino a Parigi, ha mantenuto la propria individualità senza cedere mai del tutto alla sostituzione del proprio patronimico con Dada.

Permane in territorio novecentesco il contributo di Jérôme MEIzoz (Notes sur Céline pseudonyme, pp. 83-92) sullo pseudonimo utilizzato da Louis Destouches concepito non come un tentativo di ovviare a qualsivoglia problema di censura ma come un “indicatore di posizione", un atteggiamento, l'inaugurazione di un'esistenza seconda sul piano letterario, quella di Céline appunto, complice di un gioco narrativo ambiguo, doppio dell'autore e personaggio dell'opera.

7 La seconda sezione della raccolta dal titolo «Différences identitaires», preceduta dalla breve introduzione di David Martens (Pseudonymie et différences identitaires, pp. 95-97), si apre con l'intervento di Sylvain DOURNEL dal titolo «J'habiterai mon nom»: d'Alexis Léger à Saint-John Perse, le cheminement d'un idéal (pp. 107-115). Abitare il proprio nome per Saint-John Perse significa prima di tutto darsene uno, un nome-poesia velato di sacro, non come maschera ma come proiezione di un sé ideale.

Sacro e profano nel contributo di Anne-Marie HAVARD (Jeux et enjeux de la polynomie: le cas du poète Roger Gilbert-Lecomte, pp. 117-128) in merito al caso del poeta Roger-Gilbert Lecomte, esponente di spicco del movimento del Grand Jeu. L'uso dello pseudonimo per i giovani autori del movimento rinvia ad una sorta di rinascita spirituale che ne determina l'appartenenza alla comunità vista come patria in contrapposizione a quella che viene considerata l'impostura dell'individualità.

9 La pseudonimia tira in ballo la questione della paternità per Myriam WATTHEE-DELMOTTE (De la pseudonymie comme rite autogène. La posture auctoriale de Pierre Jean Jouve et Pierre Emmanuel, pp. 129-144) che prende a esempio due scrittori considerati uno il padre spirituale dell'altro: Pierre Jean Jouve e Pierre Emmanuel. Per entrambi gli scrittori la pseudonimia sottintende motivazioni intime di ordine familiare che sanciscono l'entrata nell'ordine letterario all'insegna dell'autogenesi.

10 Segue l'articolo di Christophe MEURÉE (De l'aristocrate à l'anonyme: Marguerite Duras, pp. 145-158) in merito alle motivazioni che spinsero Marguerite Donnadieu a diventare Marguerite Duras. Christophe Meurée esplora un campo non sondato negli altri contributi: quello che lega la scelta dello pseudonimo alla sacralità della figura dello scrittore. A partire dalla possibile corrispondenza con la duchessa Claire de Duras per arrivare al linguaggio usato da Duras come simbolo dell'incontro tra proletariato e 
aristocrazia, ciò che emerge è la concezione dello scrittore come appartenente ad una "razza sacra", una "dure-race", un proletariato capace di edificare la propria nobiltà mediante la scrittura.

11 Una sottosezione è dedicata a quegli scrittori che fanno ricorso allo pseudonimo per celare o modificare l'identità culturale di appartenenza. Portando avanti uno studio sui libelli pubblicati sotto pseudonimo in occasione delle controversie religiose del XVI secolo, l'intervento di Martial MARTIN (Entre pseudonymie et anonymat. L'auctorialité brouillée des littératures satiriques et polémiques durant les guerres de religion, pp. 165-176) mostra come il nome sia in effetti segno inequivocabile d'appartenenza ad una cultura specifica.

12 Sullo stesso piano teorico si muove la riflessione di Beatrijs VANACKER (Portraits d'auteurs supposés dans quelques pseudo-traductions du xVIII siècle, pp. 177-191) che analizza il fenomeno dello pseudonimo attraverso il ricorso alla pseudo-traduzione in cui l'autore compare sotto mentite spoglie quale traduttore di presunti testi originali inglesi. Tale tendenza, pur riflettendo la forte polemica nei confronti dell'anglomania dilagante nella Francia del xviII secolo, mostra con quale cura lo pseudo-traduttore rispetti tutti gli stereotipi relativi alla cultura inglese del finto autore da lui creato.

Alle origini dello pseudonimo Joris-Karl adottato da Huysmans s'interessa l'articolo di Jérémy LAMBERT (De Georges-Charles à Joris-Karl: de la pseudonymie comme principe baptismal, pp. 193-205) il quale definisce la scelta operata dall'autore come una "trasposizione pseudonimica" e non pseudonimia vera e propria. È infatti in rispetto e devozione verso le sue origini olandesi e nel ricordo di una generazione di illustri pittori a cui sente di non appartenere del tutto che Huysmans decide di modificare il proprio nome di battesimo da Georges-Charles a Joris-Karl.

Talvolta la scelta dell'autore di ricorrere a uno pseudonimo di diversa appartenenza culturale dà origine al dibattito etico sui limiti nell'uso della pseudonimia. A questo proposito l'analisi di David MARTENS e Aleide VANMOL (Les pseudos n'ont pas tous la même peau. La réception controversée de Vernon Sullivan et Paul Smail, pp. 207-220) riporta l'esempio di Vernon Sullivan e di Paul Smaïl, pseudonimi usati da Boris Vian e JackAlain Léger nelle opere dedicate alla questione afro-americana e marocchina, accusati dalla critica di "impostura etnica".

Un'ulteriore sottosezione della seconda parte della raccolta che riflette sulle maniere in cui la pseudonimia permette all'autore di giocare sull'ambiguità di genere, si apre con l'intervento di Jean-Philippe BEAULIEU (La voix de la Maréchale d'Ancre. Effets de ventriloquie dans quelques pamphlets de 1617, pp. 229-244) sulla tecnica del ventriloquismo impiegata in alcuni pamphlets del xviII secolo a nome di Léonora Dori, la Maréchale d'Ancre, scritti presumibilmente da uomini. La tecnica del ventriloquismo come pseudonimia permette all'autore di incarnare la figura dello scriba a servizio di un supposto autore reale.

16 Assumere un'identità femminile significa in alcuni casi mobilitare una serie di stereotipi di genere per favorire l'adesione del lettore. Questo è quello che vuole mettere in luce Mélinda CARON (La "Spectatrice", "Aspasie", "La Comtesse de"... ou le masque identitaire féminin dans la presse littéraire d'Ancien Régime, pp. 245-254) studiando gli articoli pubblicati sulla stampa letteraria del xviII secolo sotto pseudonimo femminile al fine di sottolinearne l'efficacia retorica. 
17 In altri casi, l'esigenza del cambiamento di genere mediante pseudonimo è attribuito alla necessità di separare l'identità pubblica da quella letteraria oppure di rivendicare nuove forme di diffusione e di riconoscimento come spiega Sophie VAN DEN ABEELEMARCHAL (Fictions d'auteur(e)s. Pseudonymie, pseudandrie et pseudogynie entre 1825 et 1835, pp. 255-274) nella sua analisi sulle forme autoriali nella seconda metà dell'Ottocento.

Le pagine di Patricia IZQUIERDo (Jeux et enjeux de pseudonymes féminins et masculins à la Belle Époque, pp. 275-285) indagano sul travestimento onomastico durante la Belle Époque attraverso gli esempi di Yvonne de Romain, una delle prime donne a denunciare il sessismo di cui era vittima la letteratura nel primo Novecento, e di Guillaume Apollinaire che, sotto il nome di Louise Lalanne, fu l'artefice della redazione di cinque articoli a proposito della letteratura femminile per la rivista «Marges».

19 La terza e ultima sezione del volume dal titolo «Aux marges de l'auctorialité pseudonymique», è inaugurata dall'intervento di Jan HERMAN (Postures d'auteurs et doxa à l'Âge classique, pp. 293-309) sulle differenti posture dell'autore di fronte alla propria opera. Herman illustra come l'anonimato, abbondantemente utilizzato nelle opere del xviII secolo, sia strettamente legato alla pseudonimia essendo questa una delle pratiche d'elezione per preservare la propria identità.

Lo studio di Dominique MAINGUENEAU (Pseudonymie et discours constituants, pp. 311-322) concerne invece le differenti modalità di ricorso alla pseudonimia in due diverse discipline quali la letteratura e la filosofia. Se l'uso dello pseudonimo in letteratura è frequente, in un campo come la filosofia, da sempre reticente a questo determinato tipo di pratica, gli esempi sono certamente più rari. A dimostrazione di due diverse tendenze, l'articolo di Maingueneau porta l'esempio di Pierre Loti e del filosofo Alain.

21 Infine la riflessione di Charline PLUVINET (Les pseudonymes invisibles d'Éric Chevillard. «Démolir» l'autorité du nom?, pp. 323-334) analizza la tipologia di scrittura messa in atto da Éric Chevillard che, pur non firmando le sue opere con uno pseudonimo, mette in scena un tipo di scrittura volto a confondere il concetto stesso di identità, procedimento che bene si apparenta alle finalità della pratica pseudonimica. 